\title{
Ultrafast, In Situ Probing of
}

Shocked Solids at the Mesoscale and Beyond:

A New Paradigm for Materials Dynamics

Hector Lorenzana, James Belak, Geoffrey Campbell, Wayne King, Daniel Nikkel, Keith Bradley, James McNaney, John Kinney, Richard Becker, Daniel Kalantar, and Barukh Yaakobi

February $22^{\text {nd }}, 2007$

LDRD 04-ERD-071 Final Report 


\section{Disclaimer}

This document was prepared as an account of work sponsored by an agency of the United States Government. Neither the United States Government nor the University of California nor any of their employees, makes any warranty, express or implied, or assumes any legal liability or responsibility for the accuracy, completeness, or usefulness of any information, apparatus, product, or process disclosed, or represents that its use would not infringe privately owned rights. Reference herein to any specific commercial product, process, or service by trade name, trademark, manufacturer, or otherwise, does not necessarily constitute or imply its endorsement, recommendation, or favoring by the United States Government or the University of California. The views and opinions of authors expressed herein do not necessarily state or reflect those of the United States Government or the University of California, and shall not be used for advertising or product endorsement purposes.

\section{Auspices Statement}

This work was performed under the auspices of the U. S. Department of Energy (DOE) by the University of California, Lawrence Livermore National Laboratory (LLNL) under Contract No. W-7405-Eng-48. The project (04-ERD-071) was funded by the Laboratory Directed Research and Development Program at LLNL. 
UCRL-TR-228191

\title{
FY06 LDRD Final Report
}

\section{Ultrafast, I n Situ Probing of Shocked Solids at the Mesoscale and Beyond: A New Paradigm for Materials Dynamics}

\author{
LDRD Project Tracking Code: 04-ERD-071 \\ Hector Lorenzana, Principal I nvestigator
}

\begin{abstract}
Understanding material response under dynamic conditions and extreme pressures at the lattice level is important for being able to generate predictive models of material response. Despite many decades of study, material behavior is primarily inferred from bulk measurements on dynamically loaded samples or the microstructure from recovery experiments and not determined from lattice level measurements made in-situ at the relevant length scale of the governing physics. In the work described here, we report on progress made in advancing this frontier with research conducted under LDRD 04-ERD-071. Specifically, we present advances in, and applications of, dynamic x-ray diffraction, Extended X-ray Absorption Fine Structure and dynamic transmission electron microscopy.
\end{abstract}

\section{NTRODUCTI ON}

Materials dynamics, or the behavior of solids under extreme and dynamic conditions, is a topic of broad scientific and technological interest. A more mature understanding of such behavior is critical in furthering our knowledge of material performance. Of particular importance is the kinetic response of the underlying structure during shock loading, as it determines the bulk dynamic behavior. Despite this acknowledged importance, very little is known about kinetic processes under shock.

A variety of materials phenomena are manifested over multiple length and time scales during the shock response of a solid. The material responds to the shock through lattice level processes that mediate plasticity, phase transformations, damage and other phenomena as shown in Fig. 1. While the morphology and kinetics of these processes have a critical effect on the bulk

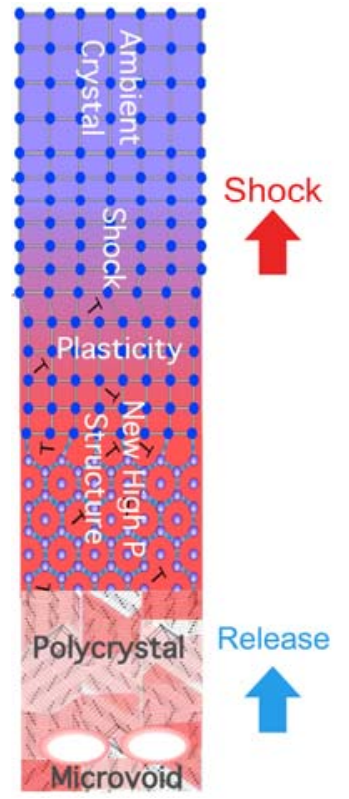

Fig 1. Schematic of a crystal under shock loading. Various material response modes can occur, and each with their own characteristic timescales. 
properties, such as strength and ductility, we still know very little about the nature of these processes.

To date two approaches have been employed to address this problem: sample recovery and bulk property measurements. In the first approach, microstructural analyses of specimens created during a shock experiment are carried out. Although this provides end-state information that can be useful in inferring dynamic behavior, the very nature of the approach does not allow direct probing of the samples under dynamic loading. This deficiency is similar to that in diamond-anvil cell experiments: while they do provide high-pressure, in situ measurements, these are essentially static studies, and cannot be expected to reflect the dynamic process.

In the second approach, measurements of the dynamic bulk response are recorded with fast diagnostics such as surface velocimetry. These approaches are clearly valuable, and in fact are primarily responsible for our current level of understanding. An example of this is Fig. 2, where the inferred kinetics and transformations are obtained from the change in slope of the wave profile. Unfortunately with this approach, the level of insight into lattice level processes is clearly limited.

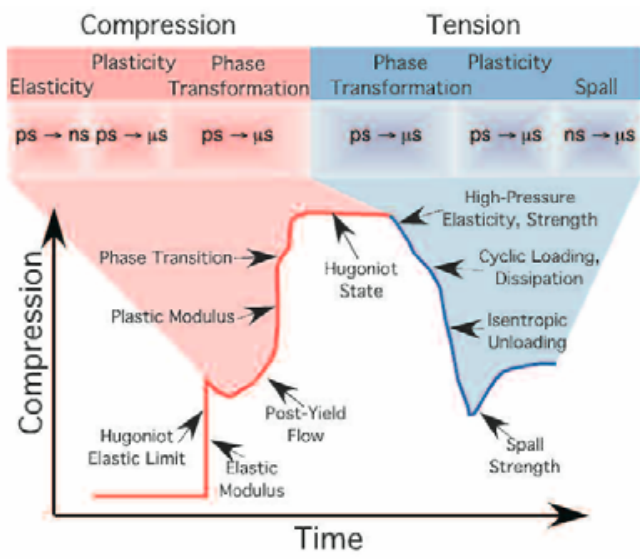

Fig 2. A shock wave profile. The mechanisms that govern the shock process span a wide range of spatial and temporal scales ${ }^{[1]}$.
To achieve a predictive understanding of material response, ultrafast diagnostic techniques are required for direct probing of the relevant transient physical processes at the lattice length scales. The necessity of this approach is illustrated by considering the metal bismuth. An overlay of the shock Hugoniot on the static phase diagram of bismuth is presented in Fig. 3. Along the Hugoniot, $\mathrm{Bi}$ has the potential for melting and resolidifying at yet higher pressures. Because of the kinetics of these processes, we cannot a priori determine the dynamic phase diagram.

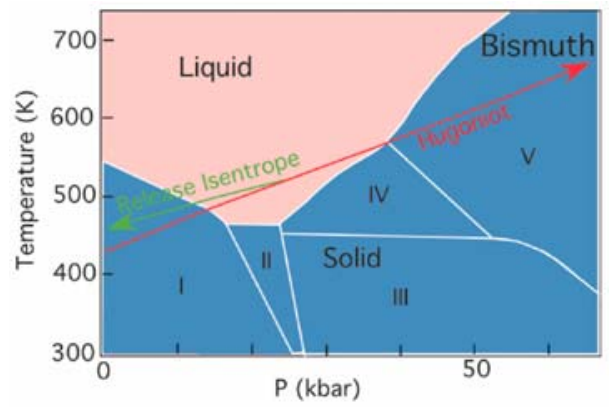

Fig 3. Static high pressure phase diagram of bismuth overlain with a shock Hugoniot. Starting at $420^{\circ} \mathrm{K}$, shocked Bi may melt and eventually resolidify. Predicting damage clearly depends on this phase history.

Prior to this work, investigations were limited to high spatial or high temporal resolution but not both, as illustrated in Fig. 4. It can be seen that the experimental techniques that allow the probing of the lattice at high spatial and temporal resolution concurrently are X-ray diffraction and scattering technologies along with dynamic electron microscopy. In this report, we focus on these three techniques, and show how their use has allowed the forging of a path into this new scientific regime of ultrafast and lattice-level scales during the shock process.

In this report, we will first present recent advancements in dynamic X-ray diffraction, using iron single crystals as an example. These are the first dynamic in situ results of the transformation from the body centered 
cubic (bcc) $\alpha$-phase to the hexagonal close packed (hcp) $\varepsilon$-phase. Iron is also used to show the ability of Extended X-ray Absorption Fine Structure (EXAFS) to elucidate the same phase transition. Lastly, the in situ, thermally driven $\alpha$ hcp to $\beta$ bcc transformation in titanium thin films is shown as observed using dynamic transmission electron microscopy (DTEM).

These studies represent the first comprehensive in situ observations of the lattice level processes that govern phase transformations and damage in the shocked solid where experimental and simulation techniques now have overlapping temporal and spatial scales.

\section{ResearCh Results}

\section{X-RAY DIFFRACTION}

One of the most studied systems at high pressure is iron, due to its technological and historical importance in society, as well as its geophysical role within the earth's core ${ }^{[2]}$. A structural transformation in iron under shock loading was inferred at about $13 \mathrm{GPa}$ based on wave profile measurements ${ }^{[3,4]}$. This was later identified as the $\alpha-\varepsilon$ phase transformation, which was observed to occur at this pressure in static experiments. Several mechanisms have been proposed for the $\alpha-\varepsilon$ transition in iron, the most common of these can be found described by Wang and Ingalls ${ }^{[5]}$.

One mechanism is shown schematically in Fig. 5. Uniaxial compression along the [001] directions places the atoms of the (110) planes into perfect hexagons by simple. A shuffling of alternate (110) planes by $a / 3 \sqrt{2}$, where $a$ is the spacing of the initial bcc lattice, establishes the atomic ordering of a pseudo-hcp lattice. In this new lattice, the c-axis corresponds to the [110] direction in the initial structure.

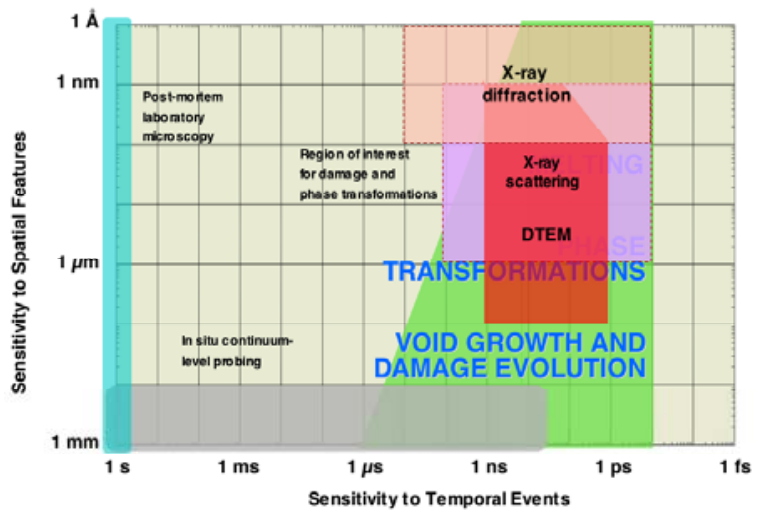

Fig 4. Spatial and temporal scales of physical processes in shocked solids as they relate to various experimental techniques.

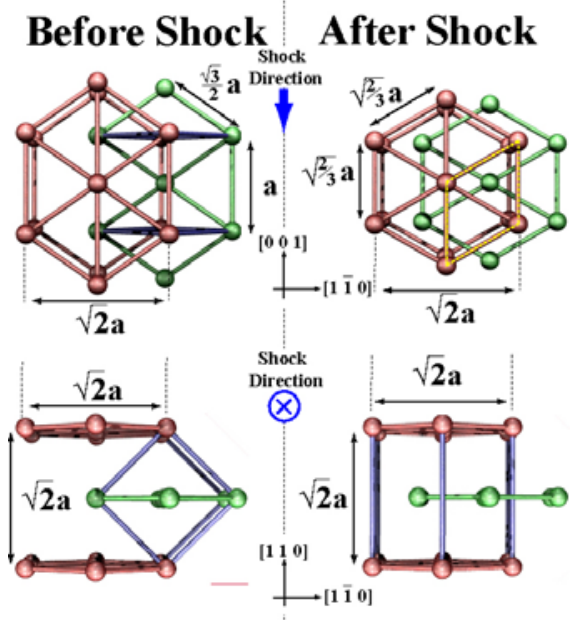

Fig 5. Schematic showing the lattice relation between $\alpha$ and $\varepsilon$ phases for iron.

The experiments were performed using the OMEGA ${ }^{[6]}$, Janus, and Vulcan ${ }^{[7]}$ lasers. Two types of sample were used: $200 \mu \mathrm{m}$ thick single crystal [001] iron obtained through Accumet and $10 \mu \mathrm{m}$ thick single crystal [001] iron fabricated by vapor deposition. A 16-20 $\mu \mathrm{m}$ parylene- $\mathrm{N}$ and 0.1 $\mu \mathrm{m}$ aluminum shine-through layer were then 
deposited on the samples to provide an ablative layer. Shock loading was achieved via direct laser irradiation at intensities ranging from $2 \times 10^{10}$ to $1 \times 10^{12} \mathrm{~W} / \mathrm{cm}^{2}$ using 2-6 ns constant intensity laser pulses.

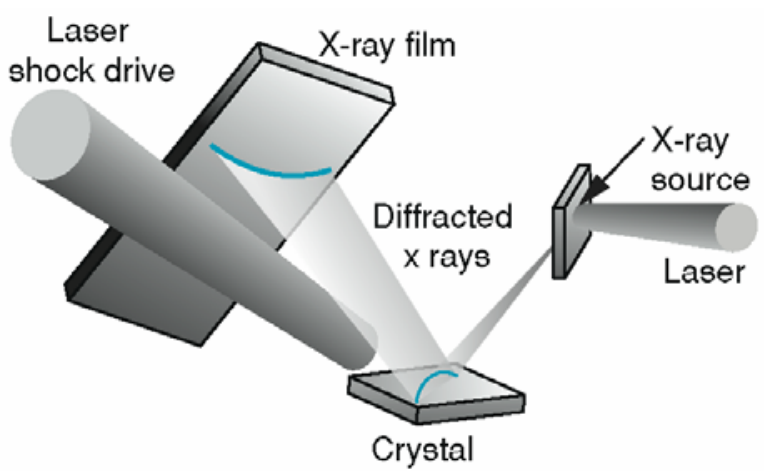

Fig 6. Geometry of the in situ wide-angle $x$-ray diffraction experiments.

Interrogation of the lattice during the shock was performed using the technique of wide-angle, in-situ diffraction ${ }^{[8,9]}$. Iron K-shell $X$-rays with a wavelength of $1.85 \AA$ were created by direct irradiation of an iron foil positioned in close proximity to the shocked crystal sample (Fig. 6). Spherically diverging $X$-rays from the source are incident on the crystal at a range of angles and planes meeting the Bragg diffraction condition produce diffracted arc features on the film. It is important to note that the Bragg condition is satisfied for some planes at locations on the crystal that are outside the region of shock loading, and as a result, not all diffraction features show compression.

Diffraction measurements of the lattice response were made at laser drive conditions that span the $\alpha-\varepsilon$ transition pressure, and Fig. 7 shows two sample diffraction images obtained from the $200 \mu \mathrm{m}$ thick samples under shock. The X-ray diffraction lines seen were diffracted in the reflection Bragg geometry from the driven surface of the crystal, and show lines from both the uncompressed and compressed lattice recorded as the shock propagated through the crystal. Fig. 7a shows diffraction from a crystal driven at low laser intensity, while Fig. 7b shows diffraction from a crystal driven at a higher intensity. In Fig. $7 a$, the diffraction lines are consistent with a uniaxial compression of the lattice by $3.9 \%$, such that the atoms are displaced solely along the shock direction. In Fig. 7b however, the shifts of the diffuse lines associated with the (002) and (101) lattice planes indicate compression of the lattice along the [001] direction by $15-18 \%$, consistent with a transformation to the hcp structure. Additionally, a shocked line that does not have a corresponding static line in Fig. $7 \mathrm{~b}$ is observed. This was identified as the $(1 \overline{1} 00)$ plane in the hcp phase.
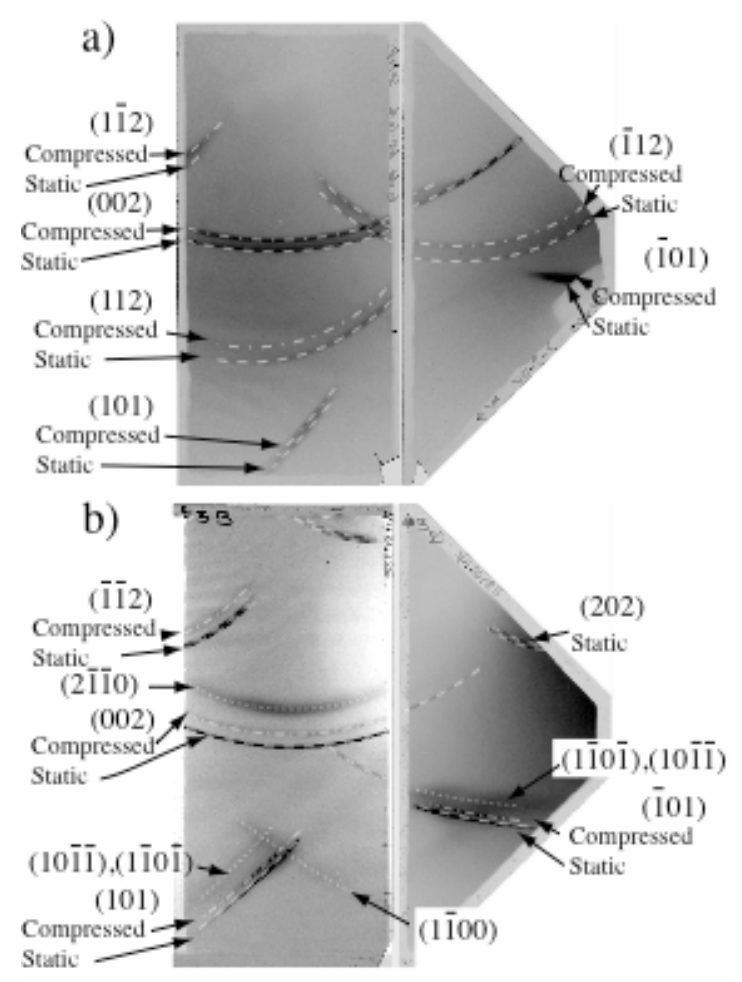

Fig 7. Sample images of the diffraction data from (a) reflection at low shock pressure, (b) reflection at high pressure. Lattice plane labels are based on the bcc and hcp coordinate systems $^{[10]}$.

Other lines that cannot be matched by diffraction from a compressed bcc lattice also appear in the transmission diffraction 


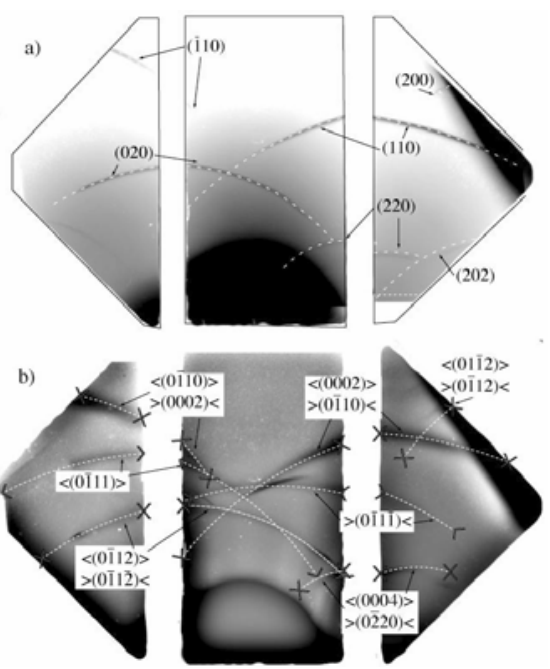

Fig 8. Transmission diffraction recorded through a $10 \mathrm{~mm}$ iron single crystal sample shocked at (a) 3.6 GPa where there is no shift in the diffraction planes perpendicular to the shock direction and (b) at 13.8GPa, above the transition threshold. images obtained from $10 \mu \mathrm{m}$ thick samples, as shown in Fig. 8. Fig. 8b shows the result of diffraction of a sample shocked above the transition pressure, and considerable broadening of the lines similar to the observations of diffraction obtained in reflection is seen. Furthermore, two new diffracted arcs are observed, corresponding to the $(0 \overline{1} 11)$ and $(0 \overline{1} 1 \overline{1})$ planes of the hcp lattice. The observation of diffraction from planes unique to the hcp lattice in both reflection and transmission provides unambiguous proof that the $\alpha-\varepsilon$ transformation in iron was observed.

\section{EXTENDED X-RAY ABSORPTION FINE STRUCTURE (EXAFS)}

The EXAFS technique possesses some unique features that make its application ideal for the experiments of interest. For example, the EXAFS spectrum of iron is markedly different in the bcc phase as compared with the high pressure hcp phase $^{[5]}$, thus providing a signature for identifying the transformation in laser-shock experiments. Additionally, in contrast to transient phase-transformation experiments that require methods for characterizing the crystal conditions (e.g., the pressure) during the transformation, or static compression experiments in which the temperature and pressure are independently controlled and measured, EXAFS allows the deduction of temperature and compression (and, hence, the pressure) directly from the captured spectra.

In this experiment, K-edge EXAFS measurements were performed on iron shocked to $35 \mathrm{GPa}$ with a 3 ns laser pulse, provided by 3 of the 60 beams of the OMEGA laser ${ }^{[6]}$. The radiation source for the EXAFS measurement was obtained by imploding a spherical target using the remaining 57 OMEGA beams. This was based on previous work ${ }^{[11]}$ that had shown that a $\mathrm{CH}$ shell imploded by a multibeam laser system emits intense and spectrally smooth radiation, lasting 120 ps. As such, this type of source is suitable for EXAFS measurements on nanosecond time scales.

In Fig. 9, we show the EXAFS spectrum measured on OMEGA for unshocked and shocked iron spectra relative to the spectra of both the bcc and hcp iron phases calculated by the FEFF8 code. The results shown in Fig. 9a assume room temperature and ambient conditions for the bcc phase, and a compression of $20 \%$ (with respect to the initial bcc density) and a temperature of $700 \mathrm{~K}$ for the hcp phase. 

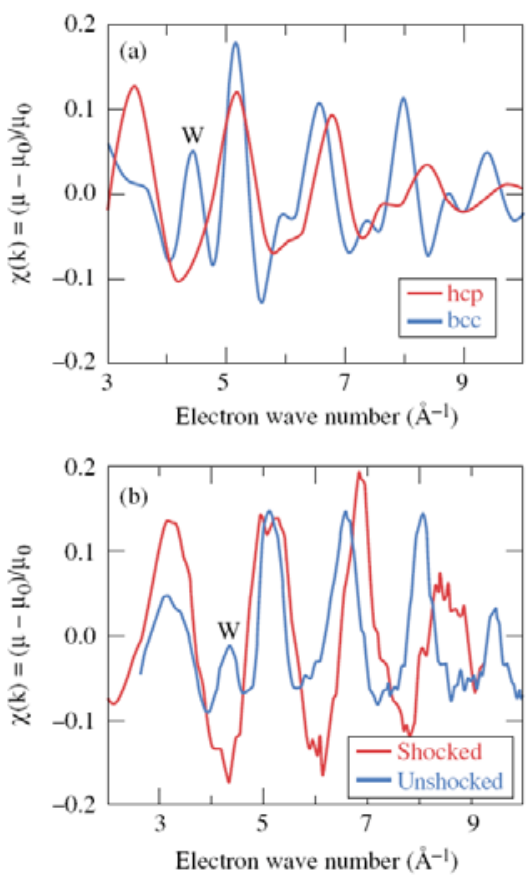

Fig 9. Spectra obtained from (a) simulation in FEFF8 for (unshocked) bcc iron and hcp iron assuming a $20 \%$ compression, (b) experimental result for unshocked and shocked iron ${ }^{[12]}$.
The main signature of the phase transformation is seen to be the disappearance of the peak marked W. When the calculations for the bcc phase were repeated for a wide range of compressions, the feature $\mathrm{W}$ remains intact. This implies that its disappearance can only be due to the phase transformation, and not due to the shock compression. Analysis of the source of the $\mathrm{W}$ indicates that it arises from a coincidence in peaks of waves scattered from the third and fourth neighboring shells in the bcc crystal. No such coincidence occurs in the hcp crystal. The only effect of compression on the EXAFS spectrum is to increase the period of oscillation (in $k$ space) and that of the heating is to cause the oscillations to decay faster with increasing $\mathrm{k}$; both are evident in Fig. 9b.

\section{DYNAMIC TRANSMISSION ELECTRON MICROSCOPY (DTEM)}

The primary focus of the DTEM work was to show that it is possible to observe a phase transformation in situ using this technique. In our specific case, the DTEM has been used to study the rapid, martensitic-type phase transformation in pure titanium films, namely, the hcp to bcc transition. In these experiments, 12 nanocrystalline titanium thin films were rapidly heated at a rate of $108 \mathrm{~K} / \mathrm{s}$ to above the transition temperature of $1155 \mathrm{~K}$. This was achieved by applying the single shot approach $^{[13]}$ and employing $1064 \mathrm{~nm}, 12 \mathrm{~ns}$ laser pulses $20-30 \mu \mathrm{J}$ covering a $0.75 \mathrm{~mm}^{2}$ spot for a power density of $3106 \mathrm{~W} / \mathrm{cm}^{2}$.

The transformation has been tracked through a series of selected area diffraction patterns collected using different delays between the pump laser heating pulse and the electron probe pulse. An example of a

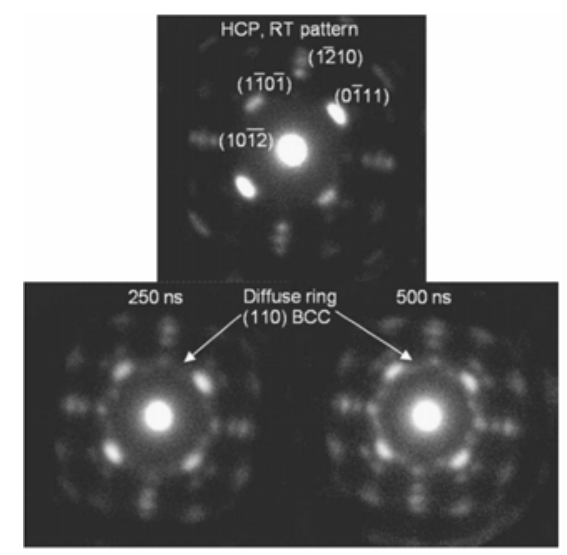

Fig 10. Electron diffraction pattern showing the evolution of the $\alpha-\beta$ transformation in $\mathrm{Ti}$ thin films with different pump/probe delays ${ }^{[14]}$.

pump/probe diffraction experiment is given in Fig. 10. The transformation initiates at 100 ns after the pump pulse, and at 250 ns after the pump laser pulse interacts with the 
specimen, the hcp phase (the stable room temperature phase) was transformed into the bcc phase as indicated by the appearance of the diffuse rings. The pattern acquired at the 500 ns delay however is significantly different from the ground-state pattern: the diffuse ring is more intense and additional spots are apparent.

The amount of bcc phase contributing to the electron diffraction can be extracted by taking the difference between the groundstate pattern and time-delayed pattern as illustrated in Fig. 11. To determine the intensities of diffraction rings, the respective difference diffraction pattern is rotationally averaged, the radial intensity with reciprocal lattice spacing is plotted, and the integrated intensities of the reflections are calculated from Gaussian fits of the peaks in the radial intensity plot. Approximate phase fractions were then calculated from a comparison of the integral intensities and by using the structure factor calculated from the JEMS software package, and the multiplicity of the reflection. The bcc phase fractions at 250 and 500 ns delays calculated from the

\section{Exit Plan}

The success of the three thrusts of this ER has ensured their continuance: the dynamic $\mathrm{x}$-ray diffraction effort has matured into a Strategic Initiative LDRD, whilst the EXAFS technique is being developed for

\section{SUMMARY}

The research effort of this LDRD resulted in three major accomplishments. The first of these is the in situ observation of the $\alpha$ to $\varepsilon$ phase transition obtained by dynamic X-ray diffraction, allowing direct observation of lattice level material behavior during the propagation of a shockwave. The second accomplishment was demonstration of how Extended X-ray Absorption Fine Structure diffraction patterns shown in Fig. 10 are $29 \pm 10$ and $50 \pm 10$ at. $\%$, respectively.

This demonstrates that the single pulse electron technique in the DTEM reveals phase transformation information at timescales on the order of $30 \mathrm{~ns}$, and that this information can be used to determine the kinetics of the transformation.

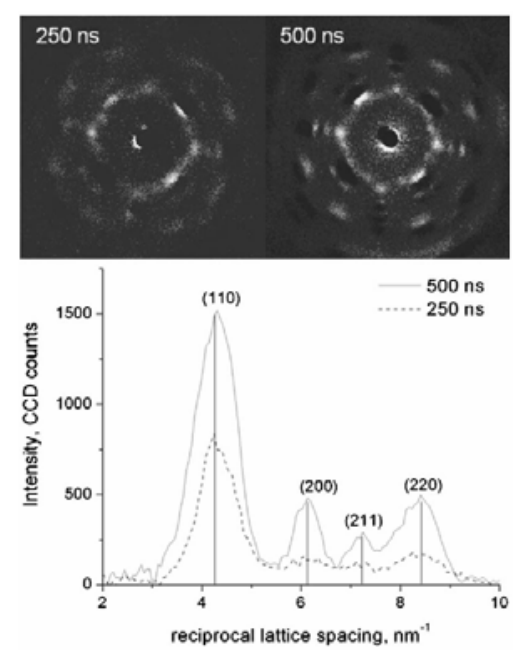

Fig 11. Difference diffraction patterns for the 250 and 500ns delays and their radial intensity distributions ${ }^{[14]}$.

applications at the National Ignition Facility. The dynamic transmission electron microscope is now a permanent facility at LLNL, with both institutional and Office of Basic Energy Sciences support.

can also be used to identify the phase transformation in iron under shock conditions. The third and final accomplishment was the in situ observation of the $\alpha$ to $\beta$ transition in titanium thin films utilizing single pulse electron pump/probes in the DTEM. 


\section{ACKNOWLEDGEMENTS}

This work was performed under the auspices of the U. S. Department of Energy (DOE) by the University of California, Lawrence Livermore National Laboratory

\section{REFERENCES}

[1] J.R. Assay, Int. J. Impact Engineering 20, 27 (1997).

[2] F. Birch, J. Geophys. Res. 57, 227 (1952).

[3] J. M. Walsh, Bull. Am. Phys. Soc. 29, 28 (1954).

[4] D. Bancroft, E. L. Peterson, and S. Minshall, J. Appl. Phys. 27, 291 (1956).

[5] F. M. Wang and R. Ingalls, Phys. Rev. $B$ 57, 5647 (1998).

[6] T. R. Boehly et al., Rev. Sci. Instrum. 66, 508 (1995).

[7] C. N. Danson et al., Opt. Commun. 103, 392 (1993).
(LLNL) under Contract No. W-7405-Eng-48. The project (04-ERD-071) was funded by the Laboratory Directed Research and Development Program at LLNL.
[8] Q. Johnson, A. Mitchell, and L. Evans, Nature 231, 310 (1971).

[9] E. Zaretsky, Appl. Phys. Lett. 78, 1 (1995).

[10] D. H. Kalantar et al., Phys. Rev. Lett. 95, 075502 (2005).

[11] B. Yaakobi et al., J. Opt. Soc. Am. B 20, 238 (2003).

[12] B. Yaakobi et al., Phys. Rev. Lett. 95, 075501 (2005).

[13] H. Dömer and O. Bostanjoglo, Rev. Sci. Instrum. 74, 4369 (2003).

[14] T. LaGrange et al., Appl. Phys. Lett. 89, 044105 (2006). 